\title{
Feasibility of Mitochondrial Transplantation Via Nose-To-Brain Delivery for Treatment of Parkinson Diseases
}

\author{
Jui-Chih Chang*1, Yi-Chun Chao ${ }^{1}$, Huei-Shin Chang ${ }^{1}$, Hui-Ju Chang ${ }^{1}$, Wen-Ling Cheng1, Ta-Tsung Lin ${ }^{1}$ and Chin-San \\ Liu*1,2,3
}

${ }^{1}$ Department of Vascular and Genomic Center, Changhua Christian Hospital, 135 Nanhsiao Street, Changhua 50094, Taiwan

${ }^{2}$ Department of Neurology, Changhua Christian Hospital, 135 Nanhsiao Street, Changhua 50094, Taiwan

${ }^{3}$ Graduate Institute of Integrated Medicine, College of Chinese Medicine, China Medical University, Taichung 40402, Taiwan

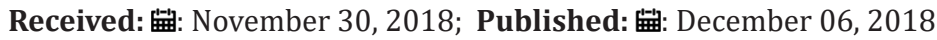

*Corresponding author: Jui-Chih Chang, Department of Vascular and Genomic Center, Changhua Christian Hospital, 135 Nanhsiao Street, Changhua 50094, Taiwan

Chin-san Liu, Department of Neurology, Changhua Christian Hospital, 135 Nanhsiao Street, Changhua 50094, Taiwan

Abbreviations: PD: Parkinson's Disease; OB: Olfactory Bulb; SN: Substantia Nigra; BBB: Blood-Brain Barrier; CNS: Central Nervous System; CSF: Cerebrospinal Fluid; BCSFB: Blood-CSF Barrier; DA: Dopamine; DOPAC: Di Hydroxy Phenyl Acetic Acid; CPP: Cell Penetrating Peptide

\section{Opinion}

Olfactory dysfunction has been recently identified as one of the earliest non-motor symptoms of Parkinson's disease (PD); it occurs at an early stage in approximately $90 \%$ of patients with PD and can be observed several years before the onset of limb disorders. Although the mechanism of olfactory dysfunction and its association with PD remain unclear, same symptoms have been observed in patients with Alzheimer disease and Down syndrome. Therefore, it is confirmed that the close association between the olfactory bulb (OB) and cranial nerves plays an important role in neurodegeneration [1]. The $\mathrm{OB}$ affects the function of dopaminergic neurons. Recent studies have demonstrated that the repeat unilateral intra-nasal administration (i.n.) of the mitochondrial inhibitor rotenone for 3-7 days can damage dopaminergic neurons in both sides of the $\mathrm{OB}$, thereby resulting in olfactory dysfunction; the inhibitory effect is more pronounced in the i.n. side. Therefore, the result suggests that rotenone affects neuronal function via olfactory transport [2].

Meanwhile, unilateral i.n. induces dopaminergic neuron damage in both sides of the $\mathrm{OB}$, indicating that neurotoxins spread to the brain via olfactory transport [2]. Therefore, i.n. may regulate brain cell functions, such as the mitochondrial functions of dopaminergic neurons via olfactory transport. Further studies confirming whether the i.n. of neurotoxins can chronically induce dopaminergic neuron death in the substantia nigra (SN) are warranted [3]; however, most studies have suggested that the delivery of inhaled environmental toxins via olfactory transport is one of the main inducers of spontaneous neurodegenerative diseases such as PD [4].

The development of effective genetic or drug therapy for PD has always been a hotspot in research. However, the efficacy of treatments is limited mainly because the delivery of therapeutic substances is hampered due to the inability of large biomolecules to cross the blood-brain barrier (BBB). One solution is to perform intra-cerebral infusion directly at the location of the lesion. In recent years, studies have confirmed the efficacy of the i.n. of nanomedicines in the treatment of PD [5]. A study found that i.n. is an effective route, which not only reduces brain tissue damage as a non-surgical method but also provides a new way to bypass the BBB via the olfactory and trigeminal neural pathways, which connect the nasal mucosa to the perivascular pathways in the brain and central nervous system (CNS) [6]. Simultaneously, the i.n. route bypasses the cardiovascular system, reduces the metabolism of drugs in the liver and increases drug utilisation, thereby providing an effective method for non-invasive drug delivery to the CNS [7]. In addition to the olfactory and trigeminal neural pathways, the study have also found that the i.n. of drugs or neurotransmitters allows these drugs to cross the nasal cavity, enter the cerebrospinal fluid (CSF) and be directly transported to the brain via the blood-CSF barrier (BCSFB) [8]. Unlike the BBB, which is formed by endothelial cells, BCSFB is located at the cerebral choroid plexus and comprises 
epithelial cells. Its functions are similar to that of the BBB, but with lower selectivity and higher permeability than the BBB to maintain CSF production by eliminating waste, maintaining neurotransmitter homeostasis and accelerating intra-cerebral substance transfer [8].

The nose-to-brain pathway enables the rapid delivery of therapeutic agents to the CNS in minutes. The rapid delivery of small and highly lipophilic molecules, whether genetic or proteinbased, into the CNS was found to have the higher efficacy via i.n. [9]. Mattern, C. et al. encapsulated the neurotransmitter dopamine (DA) in a highly lipophilic gel and used it in the treatment of PD in rats via i.n., where the encapsulation enables a regulated release of DA [9]. Their results showed that DA concentrations in the nucleus accumbens and new striatum of the rat brain increased very rapidly, thereby reaching a level that was 2-fold higher than that in the rats treated with DA aqueous solution. Moreover, DA presence was detected in the $\mathrm{OB}$, peaking at 4 hours after its administration; however, the DA presence in the treatment of non-encapsulated DA was much lower than that in treatment of lipid-encapsulated DA. In addition, DA in the CSF had not been metabolised into 3,4-dihydroxyphenylacetic acid (D0PAC) or high vanillic acid as observed normally, indicating that the use of encapsulated DA improves the efficacy of nasal DA administration in the treatment of diseases associated with DA deficiency in the brain, such as PD, attention deficit hyperactivity disorder or drug and/or alcohol addiction $[9,10]$.

The encapsulation of drugs with colloids or nanoparticles can:

a) Protect drugs from degradation during their delivery;

b) Increase drug absorption via the extracellular transfer protein P-glycoprotein and

c) Help the drug cross the olfactory epithelium. Whether the drug enters the brain via the BCSFB or trigeminal neural pathways remains unclear

d) Taken together, the successful drug administration via the nose-to-brain pathway depends on the chemical and physical properties of drugs, such as size, adhesion, stability, hydrophilicity and penetrating power. Compared with nasal inhalation, which involves the lung track, the use of nasal drops or ointments is simpler in terms of the i.n. of drugs that directly regulate brain cell function.

Mitochondria are nano-sized cell organelles; the mitochondrion of a eukaryotic cell is approximately 200-1000 nm in size. Recent animal studies have found that the in situ or intravenous injection of mitochondria isolated from healthy cells can be used in the treatment of diseases caused by mitochondrial damage. Although in vivo unmodified mitochondria may enter cells via interstitial pressure, osmosis or endocytosis $[11,12]$, the in vitro experiments performed by our research team have demonstrated that cells with mitochondrial damage, such as myoclonus epilepsy with ragged-red fibres (MERRF) syndrome, have disrupted cytoskeleton functions, thereby resulting in a decreased probability of the mitochondria entering cells via actin-dependent endocytosis [12]. To improve the efficacy of mitochondria entering the damaged cells, our team has previously studied the modification of isolated mitochondria with Pep-1, one of cell penetrating peptide (CPP) family.

Experiments on cells with mitochondrial diseases [MERRF and mitochondrial myopathy, encephalopathy, lactic acidosis, and stroke (MELAS)] [13-15] as well as animal tests in rats with PD [16] confirmed that the Pep-1-labelled mitochondria can move to the locations of damaged cell mitochondria and increase the import of mitochondria by the damaged cells to restore mitochondrial function and reduce oxidative stress. The related mechanism is associated with the inhibition of ERK phosphorylation, decrease of matrix metalloproteinases (MMP1) activation and restoration of actin cytoskeleton upstream regulatory protein cdc42 GTPase [17]. Therefore, in our recent studies we used rats with 6-OHDA drug-induced PD as models to investigate and establish a noninvasive mitochondrial transplantation system for the treatment of PD via the nose-to-brain pathway. We also explored whether Pep-1-labelling accelerates the speed of mitochondria that enter the olfactory (olfactory transport) or dopaminergic nerves through the CSF via the BCSFB and are transported to the dopaminergic neuron bodies to repair the mitochondrial function. In our previous experiments, BrdU-labelled homologous hepatic mitochondria solutions, with or without Pep-1 modification, were administrated via unilateral nasal infusion at the side of cerebral injury once a week for 3 months.

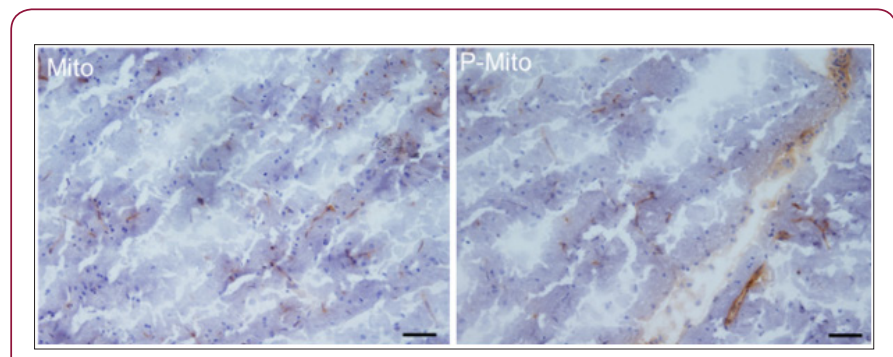

Figure 1: Expression of foreign mitochondria labelled with BrdU-labelled was observed in OB tissue (as the brown color shown) after three month grafts in PD rats. Scale bar $100 \mu \mathrm{m}$. Abbr. Mito, mitochondrial alone; P-Mito, Pep-1labelled mitochondria.

The results demonstrated that BrdU-labelled foreign mitochondria were observed both in the $\mathrm{OB}$ tissue and striatum (Figure 1). Mitochondria transplantation, with or without Pep1 modification, reduced the amphetamine-induced rotational behaviours in rats with PD. The locomotive activity of the rats, including the moving distance and rate, was also improved. The Pep-1-labelled mitochondria group (P-Mito) showed more significant improvement in locomotive activity than the Mito group [18]. Currently, the relevant pathological tissue section analysis is still in progress, including SN neuronal activity, mitochondrial function assessment, oxidative stress and the staining analysis of tyrosine hydroxylase, a marker for dopaminergic neurons. Recent studies have in fact demonstrated that stem cells may enter the CNS after i.n. [19] and can be used in the treatment of PD [20] and brain malignant tumours [21]; however, whether this approach is 
applicable to mitochondria transplantation remains unclear. Our preliminary study demonstrates the potential of nasal mitochondria delivery in treating PD as it can avoid the risk of surgery and side effects caused by invasive non-pharmacological transplantation.

\section{References}

1. Doty RL (2012) Olfactory dysfunction in Parkinson disease. Nat Rev Neurol 8(6): 329-339.

2. Sasajima H, Miyazono S, Noguchi T, Kashiwayanagi M (2015) Intranasal administration of rotenone in mice attenuated olfactory functions through the lesion of dopaminergic neurons in the olfactory bulb. Neurotoxicology 51: 106-115.

3. Lucchini R, Dorman D, Elder A, Veronesi B (2012) Neurological impacts from inhalation of pollutants and the nose-brain connection Neurotoxicology 33(4): 838-841.

4. Bove J, Perier C (2012) Neurotoxin-based models of Parkinson's disease. Neuroscience 211: 51-76.

5. Kulkarni AD, Vanjari YH, Sancheti KH, Belgamwar VS, Surana SJ, et al. (2015) Nanotechnology-mediated nose to brain drug delivery for Parkinson's disease: a mini review. J Drug Target 23(9): 775-788.

6. Jiang Y, Li Y, Liu X (2015) Intranasal delivery: circumventing the iron curtain to treat neurological disorders. Expert Opin Drug Deliv 12(11): $1717-1725$

7. Lu CT, Zhao YZ, Wong HL, Cai J, Peng L, et al. (2014) Current approaches to enhance CNS delivery of drugs across the brain barriers. Int J Nanomedicine 9: 2241-2257.

8. Laterra J, Keep R, Betz A, Goldstein G (1999) Basic neurochemistry: molecular, cellular and medical aspects. Blood-cerebrospinal fluid barrier Lippincott-Raven, Philadelphia.

9. Mattern C (2008) Controlled release delivery system for nasal application of neurotransmitters. Google Patents.

10. Kaur P, Garg T, Rath G, Goyal AK (2016) In situ nasal gel drug delivery: A novel approach for brain targeting through the mucosal membrane. Artif Cells Nanomed Biotechnol 44(4): 1167-1176.

\section{ISSN: 2574-1241}

DOI: 10.26717/BJSTR.2018.11.002158

Jui -Chih. Biomed J Sci \& Tech Res

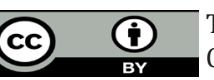

This work is licensed under Creative Commons Attribution 4.0 License

Submission Link: https://biomedres.us/submit-manuscript.php
11. Huang PJ, Kuo CC, Lee HC, Shen CI, Cheng FC, et al. (2016) Transferring xenogenic mitochondria provides neural protection against ischemic stress in ischemic rat brains. Cell Transplant 25(5): 913-927.

12. Pacak CA, Preble JM, Kondo H, Seibel P, Levitsky S, Pedro J, et al. (2015) Actin-dependent mitochondrial internalization in cardiomyocytes: evidence for rescue of mitochondrial function. Biology open 4(5): 622666.

13. Chang JC, Liu KH, Li YC, Kou SJ, Wei YH, et al. (2012) Functional recovery of human cells harbouring the mitochondrial DNA mutation MERRF A8344G via peptide-mediated mitochondrial delivery. Neurosignals 21(3-4): 160-173.

14. Chang JC, Liu KH, Chuang CS, Su HL, Wei YH, et al. (2013) Treatment of human cells derived from MERRF syndrome by peptide-mediated mitochondrial delivery. Cytotherapy 15(12): 1580-1596.

15. Chang JC, Hoel F, Liu KH, Wei YH, Cheng FC, et al. (2017) Peptidemediated delivery of donor mitochondria improves mitochondrial function and cell viability in human cybrid cells with the MELAS A3243G mutation. Sci Rep 7(1): 10710.

16. Chang JC, Wu SL, Liu KH, Chen YH, Chuang CS, et al. (2016) Allogeneic/ xenogeneic transplantation of peptide-labeled mitochondria in Parkinson's disease: restoration of mitochondria functions and attenuation of 6-hydroxydopamine-induced neurotoxicity. Transl Res 170: 40-56.

17. Liu CS, Chang JC, Kuo SJ, Liu KH, Lin TT, et al. (2014) Delivering healthy mitochondria for the therapy of mitochondrial diseases and beyond. Int J Biochem Cell Biol 53: 141-146.

18. Chang JC, Chao YC, Liu CS (2018) 15th conference of the Asian Society of Mitochondrial Research and Medicine (ASMRM 2018), Busan, Korea.

19. Jiang Y, Zhu J, Xu G, Liu X (2011) Intranasal delivery of stem cells to the brain. Opin Drug Deliv 8(5): 623-632.

20. Salama M, Sobh M, Emam M, Abdalla A, Sabry D, et al. (2017) Effect of intranasal stem cell administration on the nigrostriatal system in a mouse model of Parkinson's disease. Exp Ther Med 13(3): 976-982.

21. Li G, Bonamici N, Dey M, Lesniak MS, Balyasnikova IV (2018) Intranasal delivery of stem cell-based therapies for the treatment of brain malignancies. Opin Drug Deliv 15(2): 163-172.

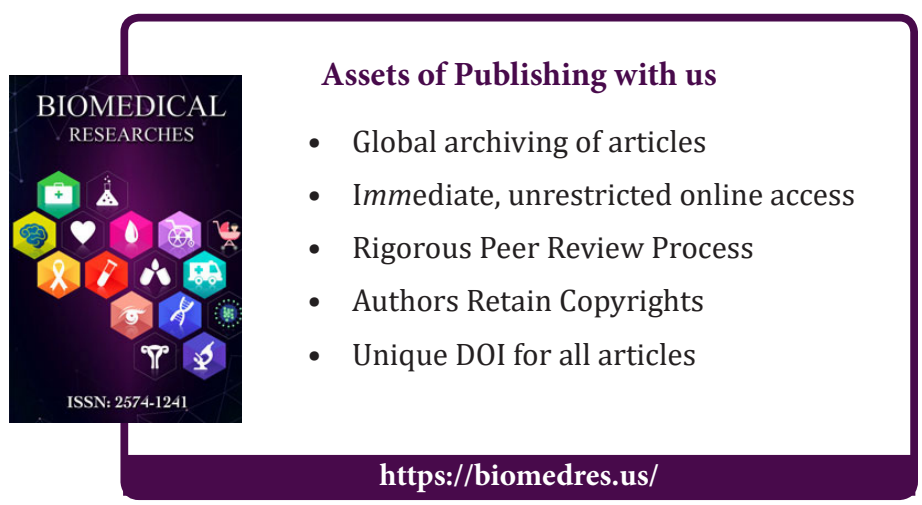

